\title{
PRODUÇÃO DA LARANJEIRA-DE-UMBIGO 'MONTE PARNASO' COM INCISÃO ANELAR DE RAMOS E USO DE REGULADORES VEGETAIS ${ }^{1}$
}

\author{
OTTO CARLOS KOLLER ${ }^{2}$, GILMAR SCHÄFER ${ }^{3}$, IVAR ANTONIO SARTORI ${ }^{3}$, \\ NESTOR VALTIR PANZENHAGEN ${ }^{3}$, JURANDIR GONÇALVES DE LIMA ${ }^{4}$
}

\begin{abstract}
RESUMO - A laranjeira-de-umbigo 'Monte Parnaso' (Citrus sinensis [L.] Osbeck) tem produtividade inferior a $10 \mathrm{~kg}$ de frutos/planta, em função de baixa fixação e/ou retenção de frutos. Com o propósito de solucionar esse problema, no ano agrícola de 2001-2002, plantas com 11 anos de idade, enxertadas sobre Poncirus trifoliata (L.) Raf., em Butiá, Rio Grande do Sul, foram submetidas aos seguintes tratamentos: 1) Testemunha: sem anelagem e sem reguladores vegetais; 2) Incisão anelar da casca dos ramos principais, 10 dias após a queda das pétalas (0210-2001); 3) Incisão anelar da casca dos ramos no final da queda natural de frutos (08-11-2001); 4) Pulverização com 5 mg.L $L^{-1}$ de ácido giberélico $\left(\mathrm{AG}_{3}\right), 10$ dias após a queda das pétalas (02-10-2001); 5) Pulverização com $15 \mathrm{mg} . \mathrm{L}^{-1}$ de ácido 2,4 - diclorofenoxiacético (2,4-D), no final da queda natural dos frutos (08-11-2001); 6) Pulverização com $50 \mathrm{mg} . \mathrm{L}^{-1}$ de ácido diclorofenoxipropiônico (2,4-DP), no final da queda natural dos frutos (08-11-2001); 7) Pulverização com 10 mg.L.-1 de $\mathrm{AG}_{3}+15$ mg.L-1 de 2,4-D, em maio (14-05-2001 e 11-05-2002); 8) Tratamentos $2+3$, e 9) Tratamentos $2+3+7$. O delineamento experimental adotado foi em blocos ao acaso, com 5 repetições, usando 3 plantas úteis por parcela. A pulverização com $5 \mathrm{mg} . \mathrm{L}^{-1}$ de $\mathrm{AG}_{3}, 10$ dias após a queda das pétalas, aumenta a produção, mas diminui a massa média dos frutos em relação a outros tratamentos que aumentam a produção, e a pulverização no final da queda natural com 15 mg.L $L^{-1}$ de $2,4-\mathrm{D}$ diminui o teor de suco dos frutos. Para aumentar a produção de frutos, basta adotar-se um dos seguintes procedimentos: em novembro, após a queda natural dos frutos, fazer a incisão anelar da casca dos ramos, ou pulverizar as plantas com $15 \mathrm{mg} . \mathrm{L}^{-1}$ de 2,4-D, ou pulverizar as plantas com 50 mg.L $\mathrm{L}^{-1}$ de 2,4-DP; ou em maio, pulverizar as plantas com $10 \mathrm{mg} . \mathrm{L}^{-1}$ de $\mathrm{AG}_{3}+15 \mathrm{mg} . \mathrm{L}^{-1}$ de 2,4-D.
\end{abstract}

Termos de indexação: Citricultura, laranja-de-umbigo, fisiologia, fitorreguladores, frutificação.

\section{FRUIT PRODUCTION OF 'MONTE PARNASO' ORANGE TREES FOLLOWING GIRDLING AND GROWTH REGULATOR SPRAYS}

\begin{abstract}
In order to reduce early fruit drop and increment fruit production of 'Monte Parnaso' oranges (Citrus sinensis [L.] Osbeck), during the season 2001/2002 orange trees from an 11 years old orchard grafted onto Poncirus trifoliata (L.) Raf. located in Butiá, Rio Grande do Sul, Brazil, were submitted to the following treatments: 1) control (no girdling and no growth regulator sprays); 2) girdling of branches after 10 days of petal drop; 3) girdling of branches after June drop; 4) $5 \mathrm{mg} . \mathrm{L}^{-1}$ of GA 3 sprayed 10 days after petal drop; 5) $15 \mathrm{mg} . \mathrm{L}^{-1}$ of 2,4-D sprayed at the end of June drop; 6) $50 \mathrm{mg} . \mathrm{L}^{-1}$ of 2,4-D sprayed at the end of June drop; 7) $10 \mathrm{mg} . \mathrm{L}^{-1}$ of GA 3 plus $15 \mathrm{mg} . \mathrm{L}^{-1}$ of 2,4-D sprayed on May $14^{\text {th }}$, 2001 and May $11^{\text {th }}, 2002 ; 8$ ) combination of treatments 2 and 3;9) combination of treatments 2,3 and 7 . The experiment was conducted in a randomized block design with 5 replicates and 3 trees as experimental units. The results indicate that spraying 5 mg. $\mathrm{L}^{-1}$ of GA 10 days after petal drop increase fruit weight production but reduce fruit average weight in relation to other treatments that enhances the fruit production; spraying $15 \mathrm{mg} . \mathrm{L}^{-1}$ of 2,4-D in November after June drop reduce fruit juice content; and to have higher fruit production one of the following procedures are recommended: girdling of branches or spraying $5 \mathrm{mg} . \mathrm{L}^{-1}$ of GA 10 days after petal drop, spraying $15 \mathrm{mg} . \mathrm{L}^{-1} \mathrm{or} 50 \mathrm{mg} . \mathrm{L}^{-1}$ of 2,4$\mathrm{D}$ in November after June drop or spraying orange trees with $10 \mathrm{mg} . \mathrm{L}^{-1}$ of GA 3 plus $15 \mathrm{mg} . \mathrm{L}^{-1}$ of 2,4-D in May.
\end{abstract}

Index terms: Citrus, navel oranges, fruit production.

\section{INTRODUÇÃO}

A citricultura é a atividade frutícola de maior destaque no Rio Grande do Sul, ocupando uma área de 42.375 ha, cuja produção de frutos gerou R $\$ 181$ milhões em 2002 (IBGE, 2004). Dentre as laranjeiras-de-umbigo, a 'Monte Parnaso' é a mais plantada, por produzir frutos de boa qualidade, grandes, com 250 a $350 \mathrm{~g}$ e maturação tardia, cuja colheita se estende de meados de agosto ao final de outubro (Koller et al., 2000), atingindo melhores preços no mercado.

Porém, nem todas as características dessa cultivar são favoráveis. O principal aspecto negativo é a baixa produtividade que, em média, nos pomares do Rio Grande do Sul, raramente ultrapassa $20 \mathrm{~kg}$ de frutos por árvore. Tem sido observado, uma exagerada abscisão de frutos novos durante a queda natural (outubro a novembro); mais tarde, de dezembro a março, geralmente após um período de 20 a 30 dias de escassez de chuvas, dependendo do ano, pode ocorrer uma segunda queda de mais de $60 \%$ dos frutos (Koller et al., 2001).

A laranjeira 'Monte Parnaso' apresenta crescimento muito vigoroso, e o florescimento é intenso, geralmente em cachos florais, muitos deles desprovidos de folhas. Esses fatores, além da ausência de sementes, são apontados por Lenz (1966), Agustí \& Almela (1991) e Becerra \& Guardiola (1984) como causadores de elevada queda de botões florais, flores e frutos, que é tanto mais intensa quanto maior o índice de floração.

O desenvolvimento dos frutos cítricos divide-se em três fases bem definidas, segundo Bain (1958): a fase I, de crescimento rápido, se estende desde a antese até o final da queda natural dos frutos, sendo esta caracterizada por intensa multiplicação celular; a fase II, também de crescimento rápido, provocado pelo aumento do tamanho das células, estende-se do final da queda natural até o início da maturação dos frutos; e a fase III, de crescimento lento, ocorre no período de maturação, até a colheita.

Todos os fatores que estimulam o crescimento do ovário ou inicial do fruto novo, como disponibilidade de nutrientes, disponibilidade hídrica e níveis hormonais (Primo-Millo, 1993) aumentam a retenção de frutos (Zucconi et al., 1978). .

Para provocar o raleio de flores e aumentar o índice de flores em ramos com folhas novas, em tangerineiras 'Clementina' e laranjeiras-de-umbigo 'Navelate', na Espanha, Agustí \& Almela (1991) recomendam pulverizar as árvores com $10 \mathrm{mg} . \mathrm{L}^{-1} \mathrm{de} \mathrm{AG}_{3}$, em novembro/dezembro (que corresponde a maio/junho no Hemisfério Sul); nesta ocasião, a adição de $15 \mathrm{mg} . \mathrm{L}^{-1}$ de 2,4-D (ácido 2,4 -

\footnotetext{
${ }^{1}$ (Trabalho 117-2005). Recebido: 27-07-2005. Aceito para publicação: 14-08-2006.

2 Doutor, Prof. Convidado da Faculdade de Agronomia da UFRGS, Bolsista 1A do CNPq. E-mail: ockoller@adufrgs.br

${ }^{3}$ Eng. Agr. Doutorando do Programa de Pós-Graduação em Fitotecnia da UFRGS

${ }^{4}$ Técnico Agrícola da Panoramas Cítrus.
} 
diclorofenoxiacético) diminui a queda de frutos no período de maturação. Na Bahia, Coelho et al. (1978) reduziram em 56\% a abscisão de laranjas 'Bahia', com pulverizações de 2,4-D e, na Argentina, Ragone (1992), aliando o uso de $\mathrm{AG}_{3}$ e 2,4-D, diminuiu a queda de laranjas-de-umbigo em $50 \%$.

Além disso, é importante pulverizar as árvores das variedades pouco produtivas, de frutos sem sementes, com $10 \mathrm{mg} . \mathrm{L}^{-1}$ de $\mathrm{AG}_{3}$ durante o florescimento (Moss, 1972), ou no final da queda das pétalas, para acelerar o crescimento dos ovários na fase I (Guardiola 1994). Esse efeito pode ser intensificado com a realização de uma incisão anelar da casca dos ramos, 10 dias após a queda das pétalas (Agustí \& Almela, 1991).

Koller et al. (2000), Koller et al. (2001) e Schäfer et al. (2001) haviam observado que se consegue aumentar em aproximadamente $50 \%$ a produção de laranjeiras 'Monte Parnaso' mediante um dos seguintes procedimentos: pulverização com $5 \mathrm{mg} . \mathrm{L}^{-1}$ de $\mathrm{AG}_{3}$, no final da queda das pétalas; anelagem da casca dos ramos principais, 10 dias após a queda das pétalas; pulverização com $15 \mathrm{mg} . \mathrm{L}^{-1} \mathrm{de}$ 2,4-D, em novembro, logo após a queda natural dos frutos; anelagem da casca dos ramos principais, em novembro, após a queda natural dos frutos. Entretanto, sem invalidar essas conclusões, em diversas situações, os referidos autores utilizaram vários procedimentos num só tratamento, como, por exemplo: aplicação de $5 \mathrm{mg} . \mathrm{L}^{-1}$ de $\mathrm{AG}_{3} \mathrm{e}$ anelagem da casca, no final da queda das pétalas $+15 \mathrm{mg} . \mathrm{L}^{-1}$ de 2,4 $\mathrm{D}$ e anelagem da casca de ramos em novembro.

Assim, este trabalho teve o objetivo principal de verificar se, mediante a aplicação de um só procedimento, com aplicação de um só fitorregulador ou anelamento da casca de ramos em cada época ou estádio fenológico. efetivamente se confirmam as respostas obtidas por Koller et al. (2000), Koller et al. (2001) e Schäfer et al. (2001), em anos anteriores.

\section{MATERIAL E MÉTODOS}

O experimento foi realizado no período de maio de 2001 a outubro de 2002, em pomar de 11 anos de idade, de laranjeiras-deumbigo 'Monte Parnaso', enxertadas sobre Poncirus trifoliata (L.) Raf., plantadas em espaçamento de 2,5 x 6,0m. O pomar está situado no Município de Butiá, distante 68 km de Porto Alegre - RS.

Foi usado um delineamento experimental em blocos ao acaso, com 9 tratamentos e 5 repetições, com 3 plantas úteis por parcela. Foram testados os seguintes tratamentos: 1) Testemunha: sem anelagem e sem fitorreguladores; 2) Anelagem da casca dos ramos principais, 10 dias após a queda das pétalas (02-10-2001); 3) Anelagem da casca dos ramos no final da queda natural de frutos $(08-11-01) ; 4)$ Pulverização com $5 \mathrm{mg} . \mathrm{L}^{-1}$ de ácido giberélico $\left(\mathrm{AG}_{3}\right), 10$ dias após a queda das pétalas (02-10-2001); 5) Pulverização com 15.mg L-1 de ácido 2,4 - diclorofenoxiacético (2,4-D), no final da queda natural dos frutos (08-11-01); 6) Pulverização com $50 \mathrm{mg} . \mathrm{L}^{-1}$ de ácido diclorofenoxipropiônico (2,4-DP), no final da queda natural dos frutos (08-11-01); 7) Pulverização com $10 \mathrm{mg} . \mathrm{L}^{-1} \mathrm{de} \mathrm{AG}_{3}+15 \mathrm{mg} . \mathrm{L}^{-1}$ de 2,4-D, em maio (14-05-01 e 11-05-02); 8) Tratamentos $2+3$, e 9) Tratamentos $2+3+7$

A fonte de $\mathrm{AG}_{3}$ foi o produto comercial PRO-GIBB ${ }^{\circledR}$ da Abbot Laboratórios do Brasil S. A. Como fonte de 2,4-D, foi usado o produto U46 ${ }^{\circledR}$ D - FLUID 2,4-D da BASF Brasileira S.A. A fonte de 2,4-DP foi através de um reagente da Sigma ${ }^{\circledR}$. Para aplicação das soluções contendo os reguladores de crescimento, foi utilizado um pulverizador de 600 litros equipado com pistolas de jato cônico direcionado, com pressão de 110 a $130 \mathrm{Lbs} / \mathrm{Pol}^{2}$.

$\mathrm{O}$ anelamento da casca foi realizado nos ramos secundários da copa, com diâmetro entre 3 e $5 \mathrm{~cm}$, fazendo incisões de $360^{\circ}$, com aproximadamente $1 \mathrm{~mm}$ de largura, sem retirar a casca utilizando uma tesoura aneladora manual, fazendo pressão suficiente para cortar a casca, mas sem danificar o lenho, conforme preconizado por Agustí \&Almela (1991).
As adubações, tratamentos fitossanitários e as demais práticas culturais foram uniformes em toda a área experimental, sendo que, nos anos anteriores, para aumentar a produtividade, as laranjeiras vinham sendo submetidas a uma pulverização com $5 \mathrm{mg}$. $\mathrm{L}^{-}$ ${ }^{1} \mathrm{de} \mathrm{AG}_{3}$, no final da queda das pétalas, e com $15 \mathrm{mg} . \mathrm{L}^{-1}$ de 2,4-D, no final da queda natural dos frutos.

O crescimento das plantas foi avaliado através da diferença existente entre o diâmetro do tronco das plantas, desde o início até o final do experimento, medido com paquímetro, numa marca previamente feita $10 \mathrm{~cm}$ acima da região de transição entre o portaenxerto e o enxerto. Por ocasião da colheita, os frutos de cada planta foram contados e pesados e, pela divisão da massa total pelo número de frutos produzidos, obteve-se a massa média dos frutos.

A percentagem de suco foi determinada relacionando a massa de suco extraído com a massa de cada amostra de frutos. A acidez total titulável (ATT) foi avaliada por titulação com hidróxido de sódio $(\mathrm{NaOH}) 0,0851 \mathrm{~N}$. Empregou-se uma amostra de $6 \mathrm{~g}$, pesada em balança semi-analítica e diluída a $50 \mathrm{ml}$ de água destilada, sob agitação constante até atingir $\mathrm{pH} 8,1$. Utilizou-se um peagâmetro Digimed DM-20, provido de um termo compensador.

O cálculo do teor de acidez foi feito de acordo com a fórmula: $\% \mathrm{~A}=\mathrm{V} * \mathrm{~N} * 0,064 * 100 / \mathrm{G}$

na qual: $\mathrm{A}=$ acidez total em gramas $\%$ de ácido cítrico; $\mathrm{N}=$ $\mathrm{NaOH}$ (normalidade); 0,064 = fator para expressar a acidez em ácido cítrico, em meq; $\mathrm{G}=$ massa da amostra de $6 \mathrm{~g}$.

O teor de SST foi obtido pingando 2 a 3 gotas de suco, retiradas da amostra homogeneizada, em um refratômetro de mão, modelo 2WAJ (Abbe Refratometer). A leitura realizada foi expressa em porcentagem (\%) de sólidos solúveis totais no suco.

A relação SST/ATT foi obtida a partir das determinações anteriormente realizadas para sólidos solúveis totais e para acidez titulável total.

Os dados obtidos foram submetidos a análises de variância, e as médias foram comparadas pelo teste de Duncan, ao nível de 5\% de probabilidade.

\section{RESULTADOS E DISCUSSÃO}

Na laranjeira 'Monte Parnaso', ocorre a queda de 70\% dos frutos desde o início até 50 dias após a queda natural (Figura 1). Depois desta fase, até a colheita, durante 300 dias, caem aproximadamente $10 \%$ dos frutos que haviam sido fixados até a queda natural.

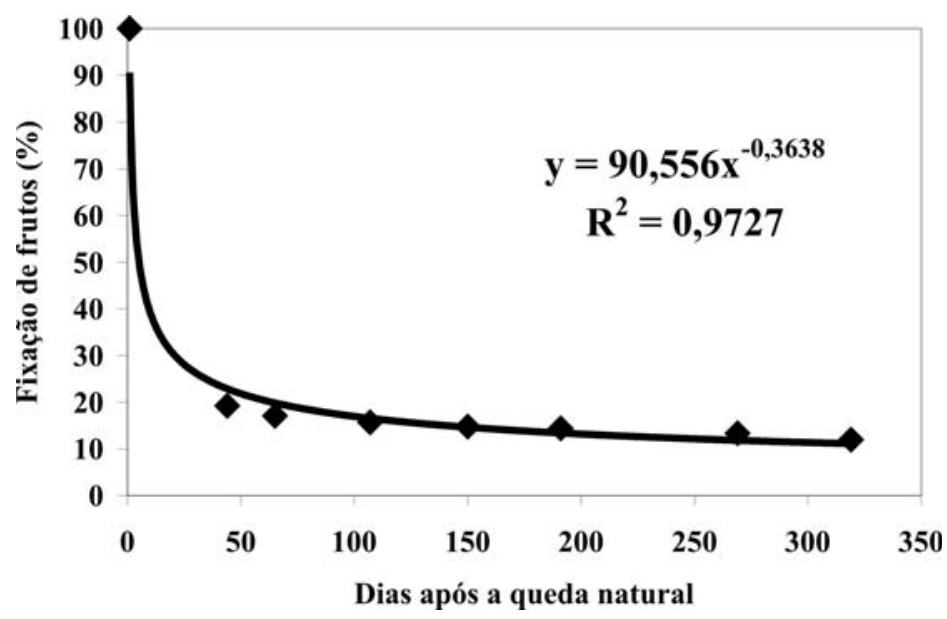

FIGURA 1 - Percentual médio de retenção de frutos, após a queda natural, em laranjeiras-de-umbigo 'Monte Parnaso' independentemente da aplicação de reguladores de crescimento e anelamento da casca de ramos. Butiá-RS, 2002. 
TABELA 1 - Número de frutos (NF), massa total de frutos (MTF) e massa média (MM) dos frutos produzidos por planta de laranjeira-deumbigo 'Monte Parnaso', com aplicação de reguladores vegetais e anelamento da casca de ramos. Butiá-RS, 2002.

\begin{tabular}{|c|c|c|c|}
\hline Tratamentos & $\mathrm{NF}$ & MTF $(\mathrm{kg})$ & $\mathrm{MM}(\mathrm{g})$ \\
\hline 1 - Testemunha & $149 b^{1}$ & $51,76 \mathrm{~b}$ & $348 \mathrm{ab}$ \\
\hline 2 - Anelagem da casca, 10 dias após a queda das pétalas & $176 \mathrm{a}$ & $59,64 \mathrm{ab}$ & $340 \mathrm{ab}$ \\
\hline 3 - Anelagem da casca, após a queda natural de frutos & $168 \mathrm{a}$ & $60,68 \mathrm{a}$ & $363 \mathrm{a}$ \\
\hline 4 - Pulverização com $5 \mathrm{mg} \cdot \mathrm{L}^{-1}$ de $\mathrm{AG}_{3}, 10$ dias após a queda de pétalas & $195 \mathrm{a}$ & $64,03 \mathrm{a}$ & $328 \mathrm{~b}$ \\
\hline 5 - Pulver. com $15 \mathrm{mg} . \mathrm{L}^{-1}$ de 2,4-D, no final da queda natural de frutos & $179 \mathrm{a}$ & 62,68 a & $351 \mathrm{ab}$ \\
\hline 6 - Pulverização com $50 \mathrm{mg} \cdot \mathrm{L}^{-1}$ de 2,4-DP, no final da queda natural frutos & $176 \mathrm{a}$ & $60,47 \mathrm{a}$ & $345 \mathrm{ab}$ \\
\hline 7 - Pulverização com $10 \mathrm{mg} \cdot \mathrm{L}^{-1} \mathrm{AG}_{3}+15 \mathrm{mg} \cdot \mathrm{L}^{-1}$ 2,4-D, em maio & $177 \mathrm{a}$ & $62,52 \mathrm{a}$ & $354 \mathrm{a}$ \\
\hline 8 - Anelagem casca, após a queda das pétalas e queda natural de frutos & $168 \mathrm{a}$ & $63,18 \mathrm{a}$ & $353 \mathrm{a}$ \\
\hline 9 - Tratamentos $2+3+7$ & $189 \mathrm{a}$ & $62,45 \mathrm{a}$ & $347 \mathrm{a}$ \\
\hline Coeficiente de variação (\%) & 10,88 & 10,10 & 4,56 \\
\hline
\end{tabular}

${ }^{1}$ Médias seguidas por letras distintas, na coluna, diferem entre si, ao nível de 5\% de significância, pelo teste de Duncan.

Com relação aos frutos colhidos, todos os tratamentos aumentaram a produção de frutos em relação à testemunha (Tabela 1), confirmando resultados anteriores obtidos por Schäfer et al. (2001) e Koller et al. (2001), de que, para aumentar a produtividade de laranjeiras-de-umbigo 'Monte Parnaso', basta utilizar um dos seguintes procedimentos: fazer o anelamento da casca dos ramos, no final da queda das pétalas, ou no final da queda natural de frutos; ou pulverizar as plantas com $5 \mathrm{mg} . \mathrm{L}^{-1} \mathrm{de} \mathrm{AG}_{3}$, no final da queda das pétalas; ou pulverizar as plantas com $15 \mathrm{mg} . \mathrm{L}^{-1}$ de 2,4-D, no final da queda natural dos frutos.

Esta resposta positiva deve estar relacionada ao aumento da divisão celular e melhor nutrição dos frutos, promovida pela aplicação de $\mathrm{AG}_{3}$ e/ou anelamento da casca dos ramos, no final da queda das pétalas, bem como pela aceleração do crescimento dos frutos, após a queda natural, promovida pelo 2,4-D, 2,4-DP e pelo anelamento da casca dos ramos (Agustí \& Almela, 1991).

Uma resposta interessante, foi que a aplicação de $10 . \mathrm{mg} \mathrm{L}^{-1}$ de $\mathrm{AG}_{3}+15 \mathrm{mg} . \mathrm{L}^{-1}$ de 2,4-D em maio (tratamento 7) também promoveu o aumento do número e da massa de frutos produzidos. Segundo Agustí \& Almela (1991) este efeito pode resultar de uma inibição da diferenciação de gemas florais, provocada pelo $\mathrm{AG}_{3}$ aplicado nesta época (Monselise \& Halevy, 1972), sendo que se o florescimento não for excessivamente abundante, haverá menor competição por nutrientes, o número proporcional de folhas novas será maior e os frutos serão melhor nutridos. Este efeito ainda não havia sido detectada em trabalhos anteriores de Koller et al. (1999a), Koller et al. (1999b), Koller et al. (2001) e Schäfer et al. (2001), porque o $\mathrm{AG}_{3}$ e o 2,4-D, aplicados no outono, sempre foram associado a outros procedimentos, como anelamento da casca, aplicação de 2,4-D e $\mathrm{AG}_{3}$ em outras épocas.

Quanto à menor massa média dos frutos verificada no tratamento 4 (com aplicação de $5 \mathrm{mg} . \mathrm{L}^{-1}$ de AG3, 10 dias antes da queda das pétalas), diferindo significativamente em relação aos tratamentos $3 ; 7 ; 8$ e 9, verifica-se que, no tratamento 4 , também ocorreu a maior produção de frutos, cujo peso médio diminuiu devido à competição que houve entre eles por nutrientes (Becerra \& Guardiola, 1984) e/ou por lenta expansão celular após a queda natural (Zucconi et al., 1978 e Guardiola, 1994).

Nos tratamentos 3; 7; 8 e 9, que aumentaram a produção, a massa média dos frutos não diminuiu porque a aplicação de auxinas (2,4-D ou 2,4-DP) e o anelamento da casca dos ramos, após a queda natural, pode ter causado a expansão das células (Agustí \& Almela,1991) e/ou melhorado a nutrição dos frutos. Contudo, a diminuição do tamanho dos frutos, que ocorreu com a aplicação de $\mathrm{AG}_{3}$ após a queda das pétalas, em função do aumento da produção, não deve ser considerada como um inconveniente, porque o peso médio de 328 gramas, para frutos de mesa, corresponde a um tamanho satisfatório.

Quanto aos índices qualitativos dos frutos, somente a percentagem de suco foi afetada pelos tratamentos (Tabela 2), sendo

TABELA 2 - Porcentagem de suco, acidez total titulável (ATT), sólidos solúveis totais (SST) e relação SST/ATT de frutos produzidos por laranjeira-de-umbigo 'Monte Parnaso' com aplicação de reguladores vegetais e anelamento da casca de ramos. Butiá-RS, 2002.

\begin{tabular}{|c|c|c|c|c|}
\hline Tratamentos & $\%$ suco & ATT & SST & SST/ATT \\
\hline 1 - Testemunha & $53,1 \mathrm{a}^{1}$ & 0,74 & 8,54 & 11,62 \\
\hline 2 - Anelagem da casca, 10 dias após a queda das pétalas & $54,1 \mathrm{a}$ & 0,78 & 8,76 & 11,36 \\
\hline 3 - Anelagem da casca, após a queda natural de frutos & $52,2 \mathrm{ab}$ & 0,77 & 8,82 & 11,54 \\
\hline 4 - Pulverização com $5 \mathrm{mg} \cdot \mathrm{L}^{-1}$ de $\mathrm{AG}_{3}, 10$ dias após a queda de pétalas & $54,1 \mathrm{a}$ & 0,73 & 8,68 & 12,08 \\
\hline 5 - Pulveriz. com $15 \mathrm{mg} \cdot \mathrm{L}^{-1}$ de 2,4-D, no final da queda natural de frutos & $50,9 \mathrm{~b}$ & 0,81 & 8,80 & 10,84 \\
\hline 6 - Pulverização com $50 \mathrm{mg} \cdot \mathrm{L}^{-1}$ de $2,4-\mathrm{DP}$, no final da queda natural frutos & $53,7 \mathrm{a}$ & 0,71 & 8,42 & 12,00 \\
\hline 7 - Pulverização com $10 \mathrm{mg} \cdot \mathrm{L}^{-1} \mathrm{AG}_{3}+15 \mathrm{mg} \cdot \mathrm{L}^{-1}$ 2,4-D, em maio & $52,7 \mathrm{ab}$ & 0,79 & 8,34 & 10,68 \\
\hline 8 - Anelagem casca, após a queda das pétalas e queda natural de frutos & $53,1 \mathrm{a}$ & 0,75 & 8,64 & 11,62 \\
\hline $9-$ Tratamentos $2+3+7$ & $52,5 \mathrm{ab}$ & 0,74 & 8,64 & 11,74 \\
\hline Coeficiente de Variação (\%) & 2,7 & 10,6 & 4,6 & 10,2 \\
\hline
\end{tabular}

${ }^{1}$ Médias seguidas por letras distintas, na coluna, diferem entre si, ao nível de 5\% de significância, pelo teste de Duncan. 
TABELA 3 - Crescimento do diâmetro do tronco de laranjeiras-de-umbigo 'Monte Parnaso' submetidas à aplicação de reguladores reguladores vegetais e anelamento da casca de ramos. Butiá-RS, 2002.

\begin{tabular}{|c|c|c|}
\hline 1 - Testemunha & $1,34 a b^{1}$ & $5,02 \mathrm{ab}$ \\
\hline 2 - Anelagem da casca, 10 dias após a queda das pétalas & $1,12 \mathrm{~b}$ & $4,04 \mathrm{~b}$ \\
\hline 3 - Anelagem da casca, após a queda natural de frutos & $1,44 \mathrm{ab}$ & $5,01 \mathrm{ab}$ \\
\hline 4 - Pulverização com $5 \mathrm{mg} \cdot \mathrm{L}^{-1}$ de $\mathrm{AG}_{3}, 10$ dias pós queda de pétalas & $1,24 \mathrm{ab}$ & $4,62 \mathrm{ab}$ \\
\hline 5 - Pulveriz. com $15 \mathrm{mg} . \mathrm{L}^{-1}$ de 2,4-D, no final da queda natural de frutos & $1,38 \mathrm{ab}$ & $4,80 \mathrm{ab}$ \\
\hline 6 - Pulveriz. com 50 mg.L $\mathrm{L}^{-1}$ de 2,4-DP, no final da queda natural frutos & $1,40 \mathrm{ab}$ & $5,04 \mathrm{ab}$ \\
\hline 7 - Pulverização com $10 \mathrm{mg} \cdot \mathrm{L}^{-1} \mathrm{AG}_{3}+15 \mathrm{mg} \cdot \mathrm{L}^{-1}$ 2,4-D, em maio & $1,66 \mathrm{a}$ & $6,03 \mathrm{a}$ \\
\hline 8 - Anelagem casca, após a queda das pétalas e queda natural de frutos & $0,92 \mathrm{~b}$ & $3,41 \mathrm{~b}$ \\
\hline $9-$ Tratamentos $2+3+7$ & $1,10 \mathrm{~b}$ & $3,92 \mathrm{~b}$ \\
\hline Coeficiente de Variação (\%) & 27,32 & 26,40 \\
\hline
\end{tabular}

${ }^{1}$ Médias seguidas por letras distintas, na coluna, diferem entre si, ao nível de $5 \%$ de significância, pelo teste de Duncan.

que, nos frutos do tratamento 5 (pulverização com $15 \mathrm{mg} . \mathrm{L}^{-1}$ de $2,4-$ $\mathrm{D}$, no final da queda natural de frutos), o teor de suco foi significativamente menor do que na testemunha e outros tratamentos, porém sem comprometer a qualidade, porque acima de $35 \%$ de suco é considerado um teor satisfatório para laranjas-deumbigo, segundo Jones et al. (1965).

Com exceção do tratamento 3 , nos tratamentos $2 ; 8$ e 9 , que envolveram a incisão anelar da casca dos ramos, ocorreu o menor crescimento do diâmetro do tronco (Tabela 3). Não houve diferença significativa em relação à testemunha e a outros tratamentos, mas isso pode ser um indício de que, favorecendo a frutificação, a anelagem da casca, aplicada durante vários anos, possa tornar mais lento o crescimento vegetativo das plantas. Isto encontraria amparo no fato de que, interrompendo temporariamente a descida da seiva elaborada, a anelagem reduz a nutrição do sistema radicular (Agustí \& Almela, 1991), prejudicando o crescimento das raízes, a absorção de água e nutrientes minerais. Além disso, aumentando a frutificação, também aumenta o consumo de nutrientes pelos frutos, em detrimento do crescimento vegetativo das plantas. Seria conveniente prolongar estudos dessa natureza, porque, se a incisão anelar da casca dos ramos efetivamente retarda o crescimento das plantas, além de aumentar a produção, essa prática teria mais um aspecto positivo, porque plantas de menor porte facilitam a colheita e a realização de tratos culturais, reduzindo custos de produção.

\section{CONCLUSÕES}

1) A produção de laranjas 'Monte Parnaso', em número e massa total de frutos, pode ser aumentada com a aplicação de uma só das seguintes práticas culturais: fazer a incisão anelar da casca dos ramos, ou pulverizar as plantas com $5 \mathrm{mg} . \mathrm{L}^{-1}$ de $\mathrm{AG}_{3}, 10$ dias após a queda das pétalas; ou fazer a incisão anelar da casca dos ramos, ou pulverizar as plantas com $15 \mathrm{mg} . \mathrm{L}^{-1}$ de 2,4-D, ou com 50 mg. $L^{-1}$ de 2,4-DP, em novembro, após a queda natural dos frutos; pulverizar as plantas com $10 \mathrm{mg} . \mathrm{L}^{-1} \mathrm{de}_{\mathrm{AG}_{3}}+15 \mathrm{mg} . \mathrm{L}^{-1} \mathrm{de}$ 2,4-D, em maio, no início da mudança de cor da casca.

2) A aplicação de $5 \mathrm{mg} . \mathrm{L}^{-1}$ de $\mathrm{AG}_{3}, 10$ dias após a queda das pétalas, aumenta a produção, mas diminui a massa média do fruto em relação a outros tratamentos que aumentam a produção, e a pulverização com $15 \mathrm{mg} \cdot \mathrm{L}^{-1}$ de 2,4-D, no final da queda natural, diminui o teor de suco dos frutos.

\section{REFERÊNCIAS}

AGUSTÍ, M.; ALMELA, V. Aplicación de fitorreguladores en citricultura. Barcelona: Aedos Editorial, 1991. 169p.
BAIN, J. M. Morphological, anatomical and physiological changes in the developing fruit of the Valencia orange, (Citrus sinensis (L.) Osbeck). Australian Journal of Botany, Victoria, v.6, p. 6976, 1958.

BECERRA, S.; GUARDIOLA, J. L. Inter-relationship between flowering and fruiting in sweet orange, cultivar Navelina. In: INTERNATIONAL CITRUS CONGRESS, 1984, São Paulo. Proceedings... Piracicaba: International Society of Citriculture, 1984. v.1, p.190-194.

COELHO, Y. S.; DUARTE, C. S.; CHITARRA, M. I. F. et al. Ácido Giberélico e 2,4-D em citros. II. Efeitos na maturação da tangerina 'Cravo' (Citrus reticulata Blanco). Revista Brasileira de Fruticultura, Cruz das Almas, v.1, n.2, p.31-44, 1978.

GUARDIOLA, J. L. Utilização de reguladores de crescimento em citricultura. Laranja, Cordeirópolis, v. 15, n. 2, p. 155-177, 1994.

IBGE. Instituto Brasileiro de Geografia e Estatística. 2002 Produção Agrícola Municipal - PAM. Disponível em: <http:// www.ibge.org.br>. Acesso: em 15 jan. 2004.

JONES, W; CREE, C.B. Enviromental factors related to fruiting of Washington Navel oranges over a 38-year period. Proceedings of the American Society Horticultural Science, Alexandria, v.86, p.267-271, 1965.

KOLLER, O. C.; FERRARI SOBRINHO, F.; SCHWARZ, S. F. Frutificação precoce de laranjeiras 'Monte Parnaso' com anelagem e pulverizações de ácido giberélico e óleo mineral. Pesquisa Agropecuária Brasileira, Brasília, v. 34, n.1, p. 63-68, 1999a.

KOLLER, O. C.; SCHÄFER, G.; SARTORI, I. A.; LIMA, J. G de. Efeito da anelagem, fitorreguladores e fungicidas sobre a fixação de frutos na laranjeira 'Monte Parnaso'. Revista Brasileira de Fruticultura, Jaboticabal - SP, v.21, n.1, p.70-73, 1999b.

KOLLER, O. C.; SCHÄFER, G.; SARTORI, I. A.; SCHWARZ, S. F.; LIMA, J. G. Produção da laranjeira-de-umbigo 'Monte Parnaso' com anelagem da casca dos ramos e aplicação de fitorreguladores. Revista Brasileira de Fruticultura, Jaboticabal, v. 22, p. 68-72, 2000. Edição especial.

KOLLER, O. C.; SCHÄFER, G.; SARTORI, I. A.; LIMA, J. G. Aumento da produtividade de laranjeiras-de-umbigo 'Monte Parnaso' com a anelagem da casca de ramos e uso de reguladores de crescimento. Laranja, Cordeirópolis, v. 22, n.2, p. 469-494, 2001.

LENZ, F. Flower and fruit development in Valencia Late orange as affected by type of inflorescence and nutritional status. Horticultural Research, Amsterdam, v.84, p.141-146, 1966.

MONSELISE, S. P.; HALEVY, A. H. Chemical inibition and promotion of citrus flower bud induction. Proceedings of the American Society Horticultural Science, Alexandria, v.84, p.514-515, 1972. 
MOSS, G. I. Promoting fruit-set and yield in sweet orange. Australian Journal Experimental Agriculture Animal and Husbandry, Collingwood,, v.12, n.54, p.96-102, 1972.

PRIMO-MILLO, E. Regulacion del cuajado del fruto en los citricos. In: CONGRESSO DECITRICULTURADE LAPLANA, 1., 1993. Anais... Valência: Ed. Ajunta de Nules, 1993. p.57-74.

RAGONE, M. L. Os reguladores de crescimento no cultivo cítrico da Argentina. In: SEMINÁRIO INTERNACIONAL DE CITROS: FISIOLOGIA, 2, Bebedouro-SP. Anais... Campinas, Fundação Cargill, p. 52-66, 1992.
SCHÄFER, G.; KOLLER, O. C.; SARTORI, I. A.; CASALI, M. E.; LIMA, J. G. Efeito de

reguladores de crescimento, aplicados em diferentes épocas e da incisão anelar dos ramos principais sobre a produção da laranjeira-de-umbigo 'Monte Parnaso'. Ciência Rural, Santa Maria, v. 31, n. 4, p. 577-581, 2001

ZUCCONI, F.; MONSELISE, S.P.; GOREN, R. Growth adscission relationship in developing orange fruit. Scientia Horticulturae, Amsterdam, v. 9, p. 137-146, 1978. 